\section{Idiopathic restrictive cardiomy- opathy - perspectives from genetics studies. Is it time to redefine these disorders?}

\author{
Ajay Bahl, Uma Nahar Saikia, \\ Madhu Khullar \\ Postgraduate Institute of Medical \\ Education and Research, Chandigarh, \\ India
}

\begin{abstract}
Idiopathic restrictive cardiomyopathy (IRC) is a rare form of heart muscle disease. Genetic studies have revealed that in about half the cases, IRC forms part of the hereditary sarcomeric contractile protein disease spectrum. Mutations in several sarcomere protein encoding genes are detected in $33-66 \%$ of cases. Among these, the mutations most commonly involve TNNI3 and MYH7. There is a disproportionately high incidence of TNNI3 mutations in patients with restrictive physiology. De novo mutations are also frequently seen in this group of patients. IRC and hypertrophic cardiomyopathy (HCM) with restrictive phenotype reflect the same or very similar disorders with different names due to arbitrary cut offs in the left ventricular wall thickness rather than two separate distinct diseases. HCM with restrictive physiology should be considered part of a continuous spectrum with IRC. This is because patients with HCM with restrictive phenotype bear far greater clinical and genetic resemblance to IRC than to rest of the HCM cohort.
\end{abstract}

\section{Introduction}

Restrictive cardiomyopathies (RCM) are a rare form of heart muscle disease. They cause heart failure and are characterized by normal or decreased volumes of both ventricles associated with biatrial enlargement, normal or near normal left ventricular wall thickness, impaired ventricular filling with restrictive physiology, and normal (or near normal) systolic function., ${ }^{1,2}$ Primary or idiopathic restrictive cardiomyopathy (IRC) is a common cause of restrictive cardiomyopathy. Two other common causes of restrictive cardiomyopathy are amyloidosis and endomyocardial fibrosis. ${ }^{3}$ Endomyocardial fibrosis though is mainly restricted to some geographical locations in the tropics like India, Africa, South and Central America.

Over the last decade, several studies have shown that sarcomeric gene mutations are an important cause of IRC. ${ }^{47}$ Also, hypertrophic cardiomyopathy (HCM) and IRC have been found to be morphologically and genetically overlapping disorders.

Use of precise parameters to define and classify any disease state is important. However, some of these may be clinically unimportant in that they may have no bearing on the course and outcome of the disease. Current definitions of restrictive cardiomyopathy and HCM have resulted in a similar confusion. ${ }^{1,2}$ These two near identical disorders differing in only one such unimportant parameter that is left ventricular wall thickness have been classified and named as different disease entities. HCM has been defined morphologically by the presence of ventricular hypertrophy while restrictive cardiomyopathy is defined physiologically by impaired ventricular filling. However, by definition restrictive cardiomyopathy should have normal left ventricular wall thickness. ${ }^{2}$ This has resulted in a peculiar situation. IRC (subset caused by sarcomeric gene mutations) and HCM with restrictive physiology though nearly identical, are classified as 2 separate disorders. Both form part of the hereditary sarcomeric contractile protein disease spectrum. HCM patients with restrictive phenotype are very similar to IRC and differ in their genetic and clinical profile from the rest of the HCM cohort. Yet, IRC is classified as RCM and HCM with restrictive phenotype is classified as HCM based only on a single parameter that is left ventricular wall thickness. This absurd situation has arisen due to the limitations in classifying disorders with overlapping features. This review will discuss the genetics of IRC and highlight the fact that HCM with restrictive physiology should be considered part of a continuous spectrum with IRC. This is because HCM with restrictive physiology bears far greater resemblance to IRC than to rest of the HCM cohort.

\section{Morphological spectrum of idiopathic restrictive cardiomyopathy}

Before discussing the genetics, it is important to understand the morphological spectrum of IRC. Like all other conditions that are labelled idiopathic, IRC is also a broad diagnosis which includes all patients in whom a specific condition cannot be found. The histo pathological changes described in a number of reports are summarized.

The most common histological finding reported in IRC patients is interstitial fibrosis and has been reported in a vast majority of patients (81-100\%). ${ }^{8-14}$ The interstial fibrosis
Correspondence: Ajay Bahl, Department of Cardiology, Postgraduate Institute of Medical Education and Research, Sector 12, Chandigarh160012, India.

Tel: +91.9815323265 - Fax: +91.172.2744401.

E-mail: drajaybahl@hotmail.com

Key words: idiopathic restrictive cardiomyopathy, hypertrophic cardiomyopathy, sarcomeric genes.

Conflict of interests: the authors declare no potential conflict of interests.

Received for publication: 30 September 2011. Revision received: 5 May 2012.

Accepted for publication: 9 May 2012.

This work is licensed under a Creative Commons Attribution NonCommercial 3.0 License (CC BYNC 3.0).

(C) Copyright A. Bahl et al., 2012

Licensee PAGEPress, Italy

Cardiogenetics 2012; 2:e4

doi:10.4081/cardiogenetics.2012.e4

can vary from mild to severe. In a large series of biopsies from 37 patients with IRC, Ammash et al. demonstrated interstitial fibrosis that was predominantly pericellular in $81 \% .^{8}$ The fibrosis was moderate to severe in 17 patients. Kaski et al. found interstitial fibrosis in all 7 hearts of patients with IRC explanted at time of transplantation. ${ }^{10}$ Some degree of endocardial fibrosis is also commonly present.

Majority of the patients also have myocyte hypertrophy. ${ }^{8-12}$ The prevalence of myocyte hypertrophy in most studies is over $80 \%{ }^{8-12}$ However in a small series of 5 patients with IRC, myocyte hypertrophy was not found in any of the endomyocardial biopsy samples. ${ }^{14}$ Some patients may show myocyte attenuation and degeneration. Myocyte vacuolation has also occasionally been reported. ${ }^{10}$ On electron microscopy no specific ultrastructural lesions are usually seen. ${ }^{9}$

An important histological feature that suggests a morphological overlap of IRC with HCM is myofibre disarray. Ammash et al did not report any myofibre disarray in biopsy samples of 37 patients with IRC. ${ }^{8}$ Other studies have commonly found myofibre disarray. ${ }^{9-12}$ This absence of myofibre disarray could be due to shortcomings of endomyocardial biopsies which unfortunately assess only tiny bits of tissue. This is exemplified in the report by Gewillig et al. ${ }^{9}$ They did not report myofibre disarray in their 4 biopsy samples. However in the three cases with full thickness examination, there was extensive myofibre disarray in the core of the myocardial wall. Myofibre disarray was thus seen in cases in whom a full heart was available but was not seen in the small biopsy tissue. In this regard the report by Kaski 
et al. is important. They reported histological findings from 7 hearts explanted at time of transplantation and thus provide a more complete picture. ${ }^{10}$ Myocyte disarray was present in 3 of their 7 cases $(42.9 \%)$.

This histological picture is very similar to that reported in patients of HCM with restrictive phenotype. Post-mortem examination in 5 of the 6 patients showed widespread myocardial disarray. ${ }^{4}$

Specific histological findings are extremely uncommon. One reported case showed hypereosinophilic inclusion bodies of unknown etiology. ${ }^{10}$ Identical findings were found in the explanted heart of this person's sister, who died after cardiac transplantation for restrictive cardiomyopathy.

Despite IRC being a broad diagnosis, the histopathological picture described in a number of case series are remarkably similar. The main histological finding found in almost all cases is a varying degree of interstitial fibrosis. Other common findings include endomyocardial fibrosis, myocyte hypertrophy, myofibre disarray and occasionally endocardial thickening (Figures 1 and 2). There are very few cases where a distinct histopathological finding is seen. A similar morphological picture seen in most cases of IRC possibly suggests similar pathophysiological basis for this disorder though this doesn't necessarily imply a common etiology. Also, histological changes resemble those of $\mathrm{HCM}$ in large number of cases with myocyte disarray being reported in around $50 \%$ of IRC patients.

\section{Clinical profile of idiopathic restrictive cardiomyopathy and hypertrophic cardiomyopathy with restrictive phenotype}

Patients with restrictive phenotype and only mild left ventricular hypertrophy form a very small but a clinically important part of the HCM cohort. Kubo et al studied a large cohort of HCM patients and found that these patients constituted only $1.5 \%$ of the cohort. ${ }^{4}$ These patients however had much worse clinical outcomes as compared to the rest of the HCM cohort. During a mean follow up of around 53 months, $32 \%$ patients died or underwent cardiac transplant. The 5-year event-free survival from any cause of death, cardiac transplantation or implantable cardioverter defibrillator discharge was only $56.4 \%$ compared to $89.8 \%$ in other HCM patients. These patients were also more symptomatic and had worse exercise capacity. Atrial fibrillation occurred in $74 \%$ patients with restrictive phenotype as compared to only $12 \%$ in rest of the HCM patients. Thus HCM patients with restrictive phenotype are much sicker and clinically very different from the rest of the HCM cohort. In fact, their clinical course is similar to that of IRC patients. In a large series of 94 mainly adult patients with IRC, $50 \%$ died during a mean follow up of 68 months and an additional 4\% underwent heart transplant. ${ }^{8}$ Poor outcomes are also seen in children with IRC. A study of 12 children (mean age 5.1 years) with IRC due to mutations in sarcomeric genes found that $75 \%$ patients underwent heart transplant and only 2 of the 12 were alive without cardiac transplant on follow up. ${ }^{10}$ Thus the clinical course of HCM with restrictive phenotype resembles that of IRC rather than that of a usual HCM cohort.

\section{Genotypic profile of idiopathic restrictive cardiomyopathy}

Mogensen et al. first reported that IRC forms part of the spectrum of hereditary sarcomeric contractile protein disease and is thus etiologically similar to HCM. ${ }^{5}$ They found mutations in the gene encoding for troponin I (TNNI3) in 6 of their nine patients with IRC.

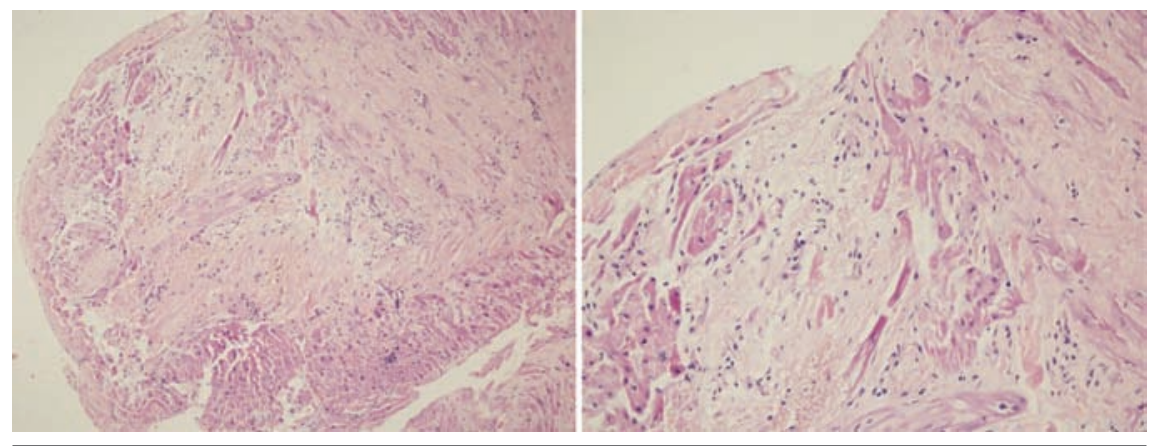

Figure 1. Microphotographs showing thickened endocardium (left) and replacement fibrosis with myocyte atrophy and anisonucleosis (right).(H \& E X 280).
The high prevalence of TNNI3 mutations in patients with IRC is indeed remarkable, considering that the prevalence of TNNI3 in an HCM cohort is only $3.1 \%{ }^{15}$ This exceptionally high prevalence of TNNI3 mutations in IRC has subsequently been confirmed in several studies.

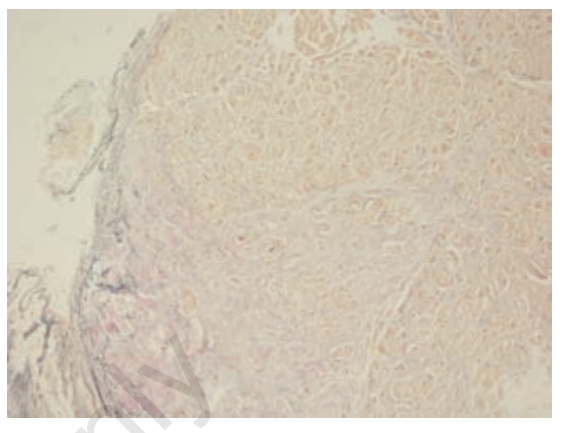

Figure 2. Microphotograph showing increased elastin in the endocardium also seen extending into the myocardium. Elastic stain X 280 .

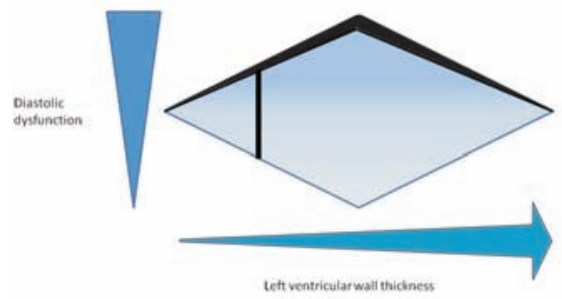

Figure 3. This figure denotes the spectrum of hypertrophic/ idiopathic restrictive cardiomyopathy. Increase in left ventricular wall thickness is denoted on the $\mathrm{X}$ axis with increasing wall thickness from left to right. In the diagonal normal left wall thickness would be on the left of the figure and severe hypertrophy on right. The vertical black line indicates the cut off between normal ventricular wall thickness on the left and left ventricular hypertrophy on the right of the line. Hypertrophic cardiomyopathy is a disorder of diastolic dysfunction. Progressive increase of diastolic dysfunction is denoted on the $\mathrm{Y}$ axis. The black band at top of the diagonal represents patients at the extreme end of diastolic dysfunction that is restrictive physiology. Patients with restrictive physiology and normal left ventricular wall thickness (to the left of the vertical black line) are labelled idiopathic restrictive cardiomyopathy. Patients with restrictive physiology and increased left ventricular wall thickness (to the right of the vertical black line) are labelled hypertrophic cardiomyopathy with restrictive physiology. 
The study by Kubo et al. provides an excellent perspective into the relationship between HCM and restrictive phenotype. ${ }^{4}$ They evaluated 1226 patients from 688 consecutive HCM families and looked for patients who had restrictive physiology with only mild left ventricular hypertrophy ( $\leq 15 \mathrm{~mm})$. One point five percent of their cohort had a restrictive phenotype. These patients were much sicker and had a worse outcome. Thus restrictive phenotype formed a very small but important part of the HCM spectrum. These patients cannot be strictly labelled IRC since they had mild left ventricular hypertrophy but fall close to the IRC phenotype. Mutations were found in 8 of the 15 families screened- 4 in TNNI3 and 4 in the gene encoding beta-myosin heavy chain (MYH7). Two of the mutations were de novo mutations. Importantly they did not find any mutation in the genes encoding cardiac myosin-binding protein $\mathrm{C}$ (MYBPC3), cardiac troponin $\mathrm{T}$ (TNNT2), and alpha-tropomyosin (TPM1). Thus the mutation profile of HCM with restrictive phenotype (high prevalence of TNNI3 mutations) is similar to that of IRC and different from that of rest of the HCM cohort.

Kaski et al. screened 12 children and found sarcomere protein gene mutations in 4 patients (33\%)- 2 in TNNI3 and 1 each in the TNNT2 and $\alpha$-cardiac actin (ACTC) genes. ${ }^{10}$ Two of these were de novo mutations.

Peddy et al. found a de novo in-frame 3 basepair deletion in TNNT2 in an infant with IRC. ${ }^{6}$ Histologic examination of the explanted heart showed significant myocyte disarray and multiple areas of replacement fibrosis. This girl also had a MYBPC3 polymorphism of unknown significance.

Rai et al. also reported a $M Y H 7$ mutation in a patient with IRC. ${ }^{7}$ They also reported a p.Arg192His mutation in TNNI3 in a adolescent Indian girl with HCM and restrictive phenotype. Two of her first degree relatives had IRC. This again affirms that HCM and IRC can be present in the same family. The same mutation has been also identified in an IRC patient of Caucasian descent with an early onset of symptoms at 16 years of age and death due to heart failure at 19 years of age. This suggests that the p.Arg192His mutation may be specifically associated with restrictive physiology. Data suggests that the expression of TNNI3 mutations is very heterogeneous and varied both within and between families with no apparent mutation or gene specific disease pattern. ${ }^{15}$ This generalization about TNNI3 mutations having heterogeneous manifestations may not apply to the p.Arg192His mutation which appears to be associated with restrictive physiology. Functional studies of this mutation in transgenic mice found that the main alteration was impaired cardiac relaxation and restrictive physiology. This manifested morphologi- cally as enlarged atria and restricted ventricles and functionally by diastolic dysfunction and diastolic heart failure. ${ }^{16}$

Desmin accumulation can also give rise to restrictive cardiomyopathy and this entity should specifically be suspected in patients who also have associated atrio-ventricular blocks. ${ }^{17,18}$ Two genes associated with desminopathy are desmin and alpha-B crystalline (CRYAB). Desmin accumulation is not evident from routine pathological examination such as hematoxylin and eosin staining of a cardiac biopsy. It can be specifically identified with immunohistochemistry. ${ }^{19}$ Mutations in desmin have been identified in these patients.

To summarize, IRC is commonly caused by mutations in sarcomere protein encoding genes (incidence of 33-66\%). The mutations most commonly involve TNNI3 and MYH7. Uncommonly mutations have also been reported in TNNT2 and ACTC. There is a disproportionately high incidence of TNNI3 mutations in patients with restrictive physiology. De novo mutations are also frequently seen in this group of patients. This is probably because of the severe phenotype and poor outcomes of these patients.

\section{What does this imply to our understanding of idiopathic restrictive cardiomyopathy?}

The availability of data on mutations found in IRC patients has shown that IRC is a disorder of mutations in sarcomeric genes. Can we thus infer that IRC is a homogeneous disorder? Presently the data is limited. Mutations in sarcomeric genes have been found in $33-66 \%$ of IRC patients. What about the rest of the patients in whom mutations have not been identified? A small number may be due to specific conditions like desminopathies while a few may have findings like inclusion bodies on histology and are likely to be due to specific disorders. The rest of the patients which would constitute around $50 \%$ of IRC patients are truly idiopathic and of unknown etiology at present. These could be due to mutations in other sarcomeric genes that have not been genotyped in this cohort or could be due to other, as yet unknown etiologies.

\section{What is in a name?}

Hypertrophic cardiomyopathy is a disorder of diastolic dysfunction. Patients with the most severe diastolic dysfunction are said to have restrictive physiology and form a very small percentage of the HCM cohort. Patients with restrictive physiology and normal left ventricular wall thickness are labelled idiopathic restrictive cardiomyopathy. Patients with restrictive physiology and increased left ventricular wall thickness are labelled hypertrophic cardiomyopathy (with restrictive physiology). Thus, HCM and IRC (at least around $50 \%$ of IRC patients) form part of a continuous disease spectrum as depicted in Figure 3. An individual with restrictive physiology and wall thickness of $12 \mathrm{~mm}$ would be labelled IRC whereas another with wall thickness of $14 \mathrm{~mm}$ would be labelled HCM with restrictive phenotype. Thus, the difference in nomenclature between IRC and HCM with restrictive phenotype only reflect the way we classify the 2 disorders using arbitrary cut offs in the left ventricular wall thickness. They appear to be the same disease rather than two separate distinct entities.

Thus HCM with restrictive phenotype should be considered part of a continuous spectrum with IRC. This is because patients having HCM with restrictive phenotype bear far greater resemblance to IRC than to rest of the HCM cohort. The classification of cardiomyopthies should reflect this clearly. Patients with HCM and restrictive phenotype need to be reclassified as part of the IRC spectrum. Lessons from genetic studies indicate that it is time to give up the criteria of normal left ventricular wall thickness in defining RCM. Patients with left ventricular hypertrophy who have restrictive physiology should also be reclassified as part of IRC disease spectrum.

\section{References}

1. Richardson P, McKenna W, Bristow M, et al. Report of the 1995 World Health Organization/International Society and Federation of Cardiology Task Force on the Definition and Classification of cardiomyopathies. Circulation 1996;93:841-2.

2. Maron BJ, Towbin JA, Thiene G, et al. Contemporary definitions and classification of the cardiomyopathies: an American Heart Association Scientific Statement from the Council on Clinical Cardiology, Heart Failure and Transplantation Committee; Quality of Care and Outcomes Research and Functional Genomics and Translational Biology Interdisciplinary Working Groups; and Council on Epidemiology and Prevention. Circulation 2006;113:1807-16.

3. Kushwaha SS, Fallon JT, Fuster V. Restrictive cardiomyopathy. N Engl J Med 1997;336:267-76.

4. Kubo T, Gimeno JR, Bahl A, et al. Prevalence, clinical significance, and 
genetic basis of hypertrophic cardiomyopathy with restrictive phenotype. J Am Coll Cardiol 2007;49:2419-26.

5. Mogensen J, Kubo T, Duque M, et al. Idiopathic restrictive cardiomyopathy is part of the clinical expression of cardiac troponin I mutations. J Clin Invest 2003;111:209-16.

6. Peddy SB, Vricella LA, Crosson JE, et al. Infantile restrictive cardiomyopathy resulting from a mutation in the cardiac troponin T gene. Pediatrics 2006;117:18303.

7. Rai TS, Ahmad S, Ahluwalia TS, et al. Genetic and clinical profile of Indian patients of idiopathic restrictive cardiomyopathy with and without hypertrophy. Mol Cell Biochem 2009;331:187-92.

8. Ammash NM, Seward JB, Bailey KR, et al. Clinical profile and outcome of idiopathic restrictive cardiomyopathy. Circulation 2000;101:2490-6.

9. Gewillig M, Mertens L, Moerman P, Dumoulin M. Idiopathic restrictive car- diomyopathy in childhood. A diastolic disorder characterized by delayed relaxation. Eur Heart J 1996;17:1413-20.

10. Kaski JP, Syrris P, Burch M, et al. Idiopathic restrictive cardiomyopathy in children is caused by mutations in cardiac sarcomere protein genes. Heart 2008;94:1478-84.

11. Hayashi T, Tsuda E, Kurosaki K, et al. Electrocardiographic and clinical characteristics of idiopathic restrictive cardiomyopathy in children. Circ J 2007;71:1534-9.

12. Russo LM, Webber SA. Idiopathic restrictive cardiomyopathy in children. Heart 2005;91:1199-202.

13. Siegel RJ, Shah PK, Fishbein MC. Idiopathic restrictive cardiomyopathy. Circulation 1984;70:165-9.

14. Hosenpud JD, Niles NR. Clinical, Hemodynamic and endomyocardial biopsy findings in idiopathic restrictive cardiomyopathy. West J Med 1986;144:303-6.

15. Mogensen J, Murphy RT, Kubo T, et al. Frequency and clinical expression of car- diac troponin I mutations in 748 consecutive families with hypertrophic cardiomyopathy. J Am Coll Cardiol 2004;44:2315-25.

16. Du J, Liu J, Feng HZ, et al. Impaired relaxation is the main manifestation in transgenic mice expressing a restrictive cardiomyopathy mutation, $\mathrm{R} 193 \mathrm{H}$, in cardiac TnI. Am J Physiol Heart Circ Physiol 2008;294:H2604-13.

17. Arbustini E, Pasotti M, Pilotto A, et al. Desmin accumulation restrictive cardiomyopathy and atrioventricular block associated with desmin gene defects. Eur J Heart Fail 2006;8:477-83.

18. Pruszczyk P, Kostera-Pruszczyk A, Shatunov A, et al. Restrictive cardiomyopathy with atrioventricular conduction block resulting from a desmin mutation. Int $\mathrm{J}$ Cardiol 2007;117:244-53.

19. Zhang J, Kumar A, Stalker HJ, et al. Clinical and molecular studies of a large family with desmin-associated restrictive cardiomyopathy. Clin Genet 2001;59:24856 . 